ESAIM: M2AN 46 (2012) 1509-1526

DOI: $10.1051 / \mathrm{m} 2 \mathrm{an} / 2012014$
ESAIM: Mathematical Modelling and Numerical Analysis

www.esaim-m2an.org

\title{
PHASE FIELD METHOD FOR MEAN CURVATURE FLOW WITH BOUNDARY CONSTRAINTS
}

\author{
Elie Bretin ${ }^{1}$ AND VAlerie PERrier ${ }^{2}$
}

\begin{abstract}
This paper is concerned with the numerical approximation of mean curvature flow $t \rightarrow \Omega(t)$ satisfying an additional inclusion-exclusion constraint $\Omega_{1} \subset \Omega(t) \subset \Omega_{2}$. Classical phase field model to approximate these evolving interfaces consists in solving the Allen-Cahn equation with Dirichlet boundary conditions. In this work, we introduce a new phase field model, which can be viewed as an Allen Cahn equation with a penalized double well potential. We first justify this method by a $\Gamma$-convergence result and then show some numerical comparisons of these two different models.
\end{abstract}

Mathematics Subject Classification. 49Q, 35B, 35K.

Received April 14, 2011. Revised January 9, 2012.

Published online June 13, 2012.

\section{INTRODUCTION}

In the last decades, a lot of work has been devoted to the motion of interfaces, and particularly to motion by mean curvature. Applications concern image processing (denoising, segmentation), material sciences (motion of grain boundaries in alloys, crystal growth), biology (modeling of vesicles and blood cells), image denoising, image segmentation and motion of grain boundaries.

Let us introduce the general setting of mean curvature flows. Let $\Omega(t) \subset \mathbb{R}^{d}, 0 \leq t \leq T$, denote the evolution by mean curvature of a smooth bounded domain $\Omega_{0}=\Omega(0)$ : the outward normal velocity $V_{n}$ at a point $x \in \partial \Omega(t)$ is given by

$$
V_{n}=\kappa,
$$

where $\kappa$ denotes the mean curvature at $x$, with the convention that $\kappa$ is negative if the set is convex. We will consider only smooth motions, which are well-defined if $T$ is sufficiently small [4]. Since singularities may develop in finite time, one may need to consider the evolution in the sense of viscosity solutions $[5,25]$.

The evolution of $\Omega(t)$ is closely related to the minimization of the following energy:

$$
J(\Omega)=\int_{\partial \Omega} 1 \mathrm{~d} \sigma .
$$

Indeed, (1.1) can be viewed as a $L^{2}$-gradient flow of this energy.

\footnotetext{
Keywords and phrases. Allen Cahn equation, mean curvature flow, boundary constraints, penalization technique, gammaconvergence, Fourier splitting method.

1 CMAP, École Polytechnique, 91128 Palaiseau, France. bretin@polytechnique.fr

2 Laboratoire Jean Kuntzmann, Université de Grenoble and CNRS, UMR 5224, B.P. 53, 38041 Grenoble Cedex 9, France.

Valerie.Perrier@imag.fr
} 
There is an important literature on numerical methods for the mean curvature flows. These can be roughly classified into three categories: parametric methods [6,7,22,23], Level set formulations [19, 24,32-34] or Phase field approaches $[11,17,31,36]$. See for instance [23] for a complete review and comparison beetween these three differents strategies.

In this work, we focus on phase field method. Following [30,31], the functional $J$ can be approximated by a Ginzburg-Landau functional:

$$
J_{\epsilon}(u)=\int_{\mathbb{R}^{d}}\left(\frac{\epsilon}{2}|\nabla u|^{2}+\frac{1}{\epsilon} W(u)\right) \mathrm{d} x,
$$

where $\epsilon>0$ is a small parameter, and $W$ is a double well potential with wells located at 0 and 1 (for example $\left.W(s)=\frac{1}{2} s^{2}(1-s)^{2}\right)$.

Modica and Mortola $[30,31]$ have shown the $\Gamma$-convergence of $J_{\epsilon}$ to $c_{W} J$ in $L^{1}\left(\mathbb{R}^{d}\right)$ (see also [9]), where

$$
c_{W}=\int_{0}^{1} \sqrt{2 W(s)} \mathrm{d} s .
$$

The corresponding Allen-Cahn equation [2], obtained as the $L^{2}$-gradient flow of $J_{\epsilon}$, reads

$$
\frac{\partial u}{\partial t}=\Delta u-\frac{1}{\epsilon^{2}} W^{\prime}(u) .
$$

Existence, uniqueness, and a comparison principle have been established for this equation (see for example Chaps. 14 and 15 in [4]). To this equation, one usually associates the profile

$$
q=\arg \min \left\{\int_{\mathbb{R}}\left(\frac{1}{2}{\gamma^{\prime}}^{2}+W(\gamma)\right) ; \gamma \in H_{l o c}^{1}(\mathbb{R}), \gamma(-\infty)=1, \gamma(+\infty)=0, \gamma(0)=\frac{1}{2}\right\} .
$$

Remark 1.1. The profile $q$ (when $W$ is continuous) can also be obtained [1] as the global decreasing solution of the following Cauchy problem

$$
\left\{\begin{array}{l}
q^{\prime}(s)=-\sqrt{W(s)}, \quad s \in \mathbb{R} \\
q(0)=\frac{1}{2},
\end{array}\right.
$$

and satisfies

$$
\int_{\mathbb{R}}\left(\frac{1}{2} q^{\prime}(s)^{2}+W(q(s))\right)=\int_{0}^{1} \sqrt{2 W(s)} \mathrm{d} s .
$$

Then, the motion $\Omega(t)$ can be approximated by

$$
\Omega_{\epsilon}(t)=\left\{x \in \mathbb{R}^{d} ; u_{\epsilon}(x, t) \geq \frac{1}{2}\right\},
$$

where $u_{\epsilon}$ is the solution of the Allen Cahn equation (1.3) with the initial condition

$$
u_{\epsilon}(x, 0)=q\left(\frac{d(x, \Omega(0))}{\epsilon}\right) .
$$

Here $d(x, \Omega)$ denotes the signed distance of a point $x$ to the set $\Omega$.

The convergence of $\partial \Omega_{\epsilon}(t)$ to $\partial \Omega(t)$ has been proved for smooth motions $[10,17]$ and in the general case without fattening [5,25]. The convergence rate has been proved to behave as $O\left(\epsilon^{2}|\log \epsilon|^{2}\right)$.

Various numericals method has been used to solve Allen Cahn equation, for instance, finite difference method $[8,20,29]$, the finite element method $[27,28,42]$. From the practical point of view, we usually solve this equation is in a box $Q$, with periodic boundary conditions, which allow solutions to be computed via a semi-implicit Fourier-spectral method as in the paper [18]. 


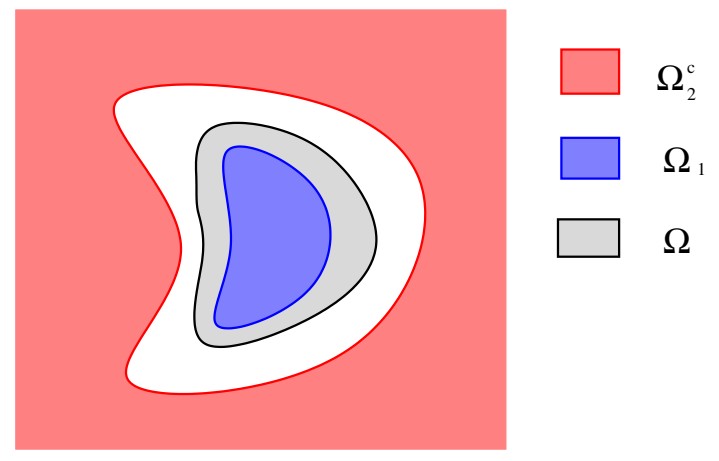

Figure 1. Constrained Mean curvature flow.

In this article, we investigate the approximation of interfaces evolving in a restricted area, which usually occurs in several physical applications. More precisely, we will consider mean curvature flow $t \rightarrow \Omega(t)$ evolving as the $L^{2}$ gradient flow of the following energy:

$$
J_{\Omega_{1}, \Omega_{2}}(\Omega)= \begin{cases}\int_{\partial \Omega} 1 \mathrm{~d} \sigma & \text { if } \Omega_{1} \subset \Omega \subset \Omega_{2}, \\ +\infty & \text { otherwise }\end{cases}
$$

Here $\Omega_{1}$ and $\Omega_{2}$ are two given smooth subsets of $\mathbb{R}^{d}$ such that $\operatorname{dist}\left(\partial \Omega_{1}, \partial \Omega_{2}\right)>0$. These evolving interfaces will clearly satisfy the following constraint $\Omega_{1} \subset \Omega(t) \subset \Omega_{2}$.

To our knowledge, the only phase field model known to approximate these evolving interfaces considers the Allen Cahn equation in $\Omega_{2} \backslash \Omega_{1}$ with Dirichlet boundary condition on $\partial \Omega_{1}$ and $\partial \Omega_{2}$ [35]. Yet, some limitations appear in this model:

- The Dirichlet boundary conditions prevent interfaces to reach boundaries $\partial \Omega_{1}$ and $\partial \Omega_{2}$. This can be seen as a consequence of thickness of the interface layer which is about $O(\epsilon \ln (\epsilon))$. This highlights the fact that the convergence rate of this model can not be better than $O(\epsilon \ln (\epsilon))$.

- From a numerical point of view, the resolution of the Allen Cahn equation with Dirichlet boundary conditions can be performed using a finite element method [26]. This choice appears more flexible to describe complex geometries, despite the computational cost associated with the construction of appropriate solution grid, especially in dimension 3. More recently, Fourier spectral discretizations have been introduced to overcome the limit choice of finite element methods in irregular domain $[16,41]$ and to produce hight-order accuracy schemes. These techniques are based on smooth immersed interfaces and penalization approach which can incorporate a large variety of boundary conditions. Note that these constructions used penalized differential operators which are ill-conditioned and should raise some instability issues in their numerical integration.

To overcome these limitations, we introduce in this paper a new phase field model. The main idea will be to consider the Allen-Cahn equation in the whole domain with a penalization technique on the double well potential $W$ to take into account the boundary constraints. Note that our approach is quite different from [16,41]. Indeed, we are not looking for some penalization technique to solve Dirichlet Allen Cahn equation but for the interface sharp-limit of a variational problem when $\epsilon$ goes to zero.

We then expect to obtain a better convergence order than $O(\epsilon \ln (\epsilon))$ for our phase field approximation. Moreover, this phase field model will be numerically solved by spectral method [18] as in [16,41]. However, our penalization terms act on the double well potential $W$, wich can be explicitely integrated without adding numerical instabililities. 
The paper is organized as follows:

In Section 2, we present in detail the two phase field model. In Section 3, we justify our penalized approach by a $\Gamma$-convergence result. In Section 4 , we compare our method to the classical Finite Element model of [35], through numerical illustrations. These simulations will investigate the numerical convergence rate of each model.

\section{Phase field MOdel With Boundary CONSTRAints}

In this section, we will introduce our Allen Cahn model for the approximation of mean curvature flow $t \rightarrow \Omega(t)$ evolving as the $L^{2}$ gradient flow of the following energy

$$
J_{\Omega_{1}, \Omega_{2}}(\Omega)= \begin{cases}\int_{\partial \Omega} 1 \mathrm{~d} \sigma & \text { if } \Omega_{1} \subset \Omega \subset \Omega_{2} \\ +\infty & \text { otherwise }\end{cases}
$$

where $\Omega_{1}$ and $\Omega_{2}$ are two given smooth subsets of $\mathbb{R}^{d}$ satisfying $\operatorname{dist}\left(\partial \Omega_{1}, \partial \Omega_{2}\right)>0$. We begin with the description of the classical approach.

\subsection{Classical model with Dirichlet boundary conditions}

The classical strategy, see for instance $[12,35]$, consists in introducing the function space

$$
X_{\Omega_{1}, \Omega_{2}}=\left\{u \in H^{1}\left(\Omega_{2} \backslash \Omega_{1}\right) ; u_{\mid \partial \Omega_{1}}=1 \quad, u_{\mid \partial \Omega_{2}}=0\right\},
$$

and a penalized Ginzburg-Landau energy of the form

$$
\tilde{J}_{\epsilon, \Omega_{1}, \Omega_{2}}(u)= \begin{cases}\int_{\Omega_{2} \backslash \Omega_{1}}\left(\frac{\epsilon}{2}|\nabla u|^{2}+\frac{1}{\epsilon} W(u)\right) \mathrm{d} x & \text { if } u \in X_{\Omega_{1}, \Omega_{2}} \\ +\infty & \text { otherwise. }\end{cases}
$$

In such framework, Chambolle and Bourdin [12] have shown the $\Gamma$-convergence of $\tilde{J}_{\epsilon, \Omega_{1}, \Omega_{2}}$ to $c_{W} J_{\Omega_{1}, \Omega_{2}}$ in $L^{1}\left(\mathbb{R}^{d}\right)\left(c_{W}\right.$ has been introduced in (1.2)). This approximation conduces to the following Allen-Cahn equation

$$
\left\{\begin{array}{l}
u_{t}=\triangle u-\frac{1}{\epsilon^{2}} W^{\prime}(u), \quad \text { on } \quad \Omega_{2} \backslash \Omega_{1} \\
u_{\mid \partial \Omega_{1}}=1, \quad u_{\mid \partial \Omega_{2}}=0 \\
u(0, x)=u_{0} \in X_{\Omega_{1}, \Omega_{2}} .
\end{array}\right.
$$

A more general $\Gamma$-convergence result for the Allen-Cahn equation with Dirichlet boundary conditions can be found in [35].

\subsection{Novel approach with a penalized double well potential}

Now, we describe an alternative approach to force the boundary constraints, based on a penalized double well potential. From $W$ considered in section 1.1, we define two continuous and positive potentials $W_{1}$ and $W_{2}$ satisfying the following assumption:

$$
(H 1)\left\{\begin{array} { l l } 
{ W _ { 1 } ( s ) = W ( s ) } & { \text { for } s \geq 1 / 2 } \\
{ W _ { 1 } ( s ) \geq \operatorname { m a x } ( W ( s ) , \lambda ) } & { \text { for } s \leq 1 / 2 }
\end{array} \text { and } \quad \left\{\begin{array}{ll}
W_{2}(s)=W(s) & \text { for } s \leq 1 / 2 \\
W_{2}(s) \geq \max (W(s), \lambda) & \text { for } s \geq 1 / 2
\end{array}\right.\right.
$$

for a given constant $\lambda>0$.

For $\alpha>1, \epsilon>0$, and $x \in \mathbb{R}^{d}$, we introduce also a penalized double well potential $W_{\epsilon, \Omega_{1}, \Omega_{2}, \alpha}$ defined by

$$
\begin{aligned}
W_{\epsilon, \Omega_{1}, \Omega_{2}, \alpha}(s, x)= & W_{1}(s) q\left(\frac{\operatorname{dist}\left(x, \Omega_{1}\right)}{\epsilon^{\alpha}}\right)+W_{2}(s) q\left(\frac{\operatorname{dist}\left(x, \Omega_{2}^{c}\right)}{\epsilon^{\alpha}}\right) \\
& +W(s)\left(1-q\left(\frac{\operatorname{dist}\left(x, \Omega_{1}\right)}{\epsilon^{\alpha}}\right)-q\left(\frac{\operatorname{dist}\left(x, \Omega_{2}^{c}\right)}{\epsilon^{\alpha}}\right)\right),
\end{aligned}
$$


where $\operatorname{dist}\left(x, \Omega_{1}\right)$ and $\operatorname{dist}\left(x, \Omega_{2}^{c}\right)$ are respectively the signed distance functions to the sets $\Omega_{1}$ and $\Omega_{2}^{c}$, and $q$ is the profile function associated to $W$ defined in (1.4).

Our modified Ginzburg-Landau energy $J_{\epsilon, \Omega_{1}, \Omega_{2}, \alpha}$ reads

$$
J_{\epsilon, \Omega_{1}, \Omega_{2}, \alpha}(u)=\int_{\mathbb{R}^{d}}\left[\frac{\epsilon}{2}|\nabla u|^{2}+\frac{1}{\epsilon} W_{\epsilon, \Omega_{1}, \Omega_{2}, \alpha}(u, x)\right] \mathrm{d} x .
$$

We will prove in the next section that this energy $\Gamma$-converges to $c_{W} J_{\Omega_{1}, \Omega_{2}}$. The associated Allen-Cahn equation reads in this context:

$$
\partial_{t} u=\triangle u-\frac{1}{\epsilon^{2}} \partial_{s} W_{\epsilon, \Omega_{1}, \Omega_{2}, \alpha}(u, x) .
$$

\section{Approximation Result of the penalized GinZBurg-Landau energy}

In this section, we prove the convergence of the Ginzburg-Landau energy $J_{\epsilon, \Omega_{1}, \Omega_{2}, \alpha}$ introduced in (2.2), to the following penalized perimeter

$$
J_{\Omega_{1}, \Omega_{2}}(u)= \begin{cases}|D u|\left(\mathbb{R}^{d}\right) & \text { if } u=\mathbb{1}_{\Omega} \text { and } \Omega_{1} \subset \Omega \subset \Omega_{2} \\ +\infty & \text { otherwise. }\end{cases}
$$

Remark 3.1. Given $u \in L^{1}\left(\mathbb{R}^{d}\right),|D u|\left(\mathbb{R}^{d}\right)$ is defined by

$$
|D u|\left(\mathbb{R}^{d}\right)=\sup \left\{\int_{\mathbb{R}^{d}} u \operatorname{div}(g) \mathrm{d} x ; g \in C_{c}^{1}\left(\mathbb{R}^{d}, \mathbb{R}^{d}\right)\right\},
$$

where $C_{c}^{1}\left(\mathbb{R}^{d} ; \mathbb{R}^{d}\right)$ is the set of $C^{1}$ vector functions from $\mathbb{R}^{d}$ to $\mathbb{R}^{d}$ with compact support in $\mathbb{R}^{d}$. If $u \in W^{1,1}\left(\mathbb{R}^{d}\right)$, $|D u|$ coincides with the $L^{1}$-norm of $\nabla u$ and if $u=\mathbb{1}_{\Omega}$ where $\Omega$ has a smooth boundary, $|D u|$ coincides with the perimeter of $\Omega$. Moreover, $u \rightarrow|D u|\left(\mathbb{R}^{d}\right)$ is lower semi-continuous in $L^{1}\left(\mathbb{R}^{d}\right)$ topology.

We assume in this section that $\Omega_{1}$ and $\Omega_{2}$ are two given smooth subsets of $\mathbb{R}^{d}$ satisfying $\operatorname{dist}\left(\partial \Omega_{1}, \partial \Omega_{2}\right)>0$, and that $\epsilon$ is sufficiently small such that

$$
1-q\left(\frac{\operatorname{dist}\left(x, \Omega_{1}\right)}{\epsilon^{\alpha}}\right)-q\left(\frac{\operatorname{dist}\left(x, \Omega_{2}^{c}\right)}{\epsilon^{\alpha}}\right)>1 / 2,
$$

for all $x$ in $\Omega_{2} \backslash \Omega_{1}$.

We now state the main result for the modified Ginzburg-Landau energy $J_{\epsilon, \Omega_{1}, \Omega_{2}, \alpha}$ :

Theorem 3.2. Assume that $W$ is a positive double-well potential with wells located at 0 and 1 , continuous on $\mathbb{R}$ and such that $W(s)=0$ if and only if $s \in\{0,1\}$. Assume also that $W_{1}$ and $W_{2}$ are two continuous potentials satisfying assumption ( $H 1)$. Then, for any $\alpha>1$, it holds

$$
\Gamma-\lim _{\epsilon \rightarrow 0} J_{\epsilon, \Omega_{1}, \Omega_{2}, \alpha}=c_{W} J_{\Omega_{1}, \Omega_{2}} \quad \text { in } \quad L^{1}\left(\mathbb{R}^{d}\right) .
$$

Proof. We first prove the liminf inequality.

(i) Liminf inequality:

Let $\left(u_{\epsilon}\right)$ converge to $u$ in $L^{1}\left(\mathbb{R}^{d}\right)$. As $J_{\epsilon, \Omega_{1}, \Omega_{2}, \alpha} \geq 0$, it is not restrictive to assume that the liminf of $J_{\epsilon, \Omega_{1}, \Omega_{2}}\left(u_{\epsilon}\right)$ is finite. So we can extract a subsequence $u_{n}=u_{\epsilon_{n}}$ such that

$$
\lim _{n \rightarrow+\infty} J_{\epsilon_{h}, \Omega_{1}, \Omega_{2}, \alpha}\left(u_{n}\right)=\liminf _{\epsilon \rightarrow 0} J_{\epsilon, \Omega_{1}, \Omega_{2}, \alpha}\left(u_{\epsilon}\right) \in \mathbb{R}^{+} .
$$


Note that from Remark (1.1) and assumption (3.1), it holds

$$
\begin{cases}q\left(\frac{\operatorname{dist}\left(x, \Omega_{1}\right)}{\epsilon^{\alpha}}\right) \geq 1 / 2 & \text { for } x \in \Omega_{1}, \\ q\left(\frac{\operatorname{dist}\left(x, \Omega_{2}^{c}\right)}{\epsilon^{\alpha}}\right) \geq 1 / 2 & \text { for } x \in \Omega_{2}^{c}, \\ 1-q\left(\frac{\operatorname{dist}\left(x, \Omega_{1}\right)}{\epsilon^{\alpha}}\right)-q\left(\frac{\operatorname{dist}\left(x, \Omega_{2}^{c}\right)}{\epsilon^{\alpha}}\right) \geq 1 / 2 & \text { for } x \in \Omega_{2} \backslash \Omega_{1}, \\ 1-q\left(\frac{\operatorname{dist}\left(x, \Omega_{1}\right)}{\epsilon^{\alpha}}\right)-q\left(\frac{\operatorname{dist}\left(x, \Omega_{2}^{c}\right)}{\epsilon^{\alpha}}\right) \geq 0 & \text { for } x \in \mathbb{R}^{d},\end{cases}
$$

for $\epsilon$ sufficiently small.

This implies that

$$
\begin{aligned}
\int_{\Omega_{1}} W_{1}\left(u_{n}\right) \mathrm{d} x & \leq \int_{\Omega_{1}} 2 q\left(\frac{\operatorname{dist}\left(x, \Omega_{1}\right)}{\epsilon_{n}^{\alpha}}\right) W_{1}\left(u_{n}\right) \mathrm{d} x \\
& \leq 2 \int_{\mathbb{R}^{d}} W_{\epsilon_{n}, \Omega_{1}, \Omega_{2}, \alpha}\left(u_{n}, x\right) \mathrm{d} x \\
& \leq 2 \epsilon_{h} J_{\epsilon_{n}, \Omega_{1}, \Omega_{2}, \alpha}\left(u_{n}\right) .
\end{aligned}
$$

In the same way:

$$
\int_{\mathbb{R}^{d} \backslash \Omega_{2}} W_{2}\left(u_{n}\right) \mathrm{d} x \leq 2 \epsilon_{n} J_{\epsilon_{n}, \Omega_{1}, \Omega_{2}, \alpha}\left(u_{n}\right) \quad \text { and } \quad \int_{\Omega_{2} \backslash \Omega_{1}} W\left(u_{n}\right) \mathrm{d} x \leq 2 \epsilon_{n} J_{\epsilon_{n}, \Omega_{1}, \Omega_{2}, \alpha}\left(u_{n}\right) .
$$

At the limit $n \rightarrow \infty$, the Fatou's Lemma and the continuity of $W, W_{1}$ and $W_{2}$ imply that $\int_{\Omega_{1}} W_{1}(u) \mathrm{d} x=0$, $\int_{\mathbb{R}^{d} \backslash \Omega_{2}} W_{2}(u) \mathrm{d} x=0$ and $\int_{\Omega_{2} \backslash \Omega_{1}} W(u) \mathrm{d} x=0$. Recall also that $W, W_{1}$ and $W_{2}$, vanish respectively at $s=\{0,1\}$, $s=\{1\}$ and $s=\{0\}$. This means that

$$
u(x) \in \begin{cases}\{1\} & \text { a.e in } \Omega_{1} \\ \{0\} & \text { a.e in } \mathbb{R}^{d} \backslash \Omega_{2} \\ \{0,1\} & \text { a.e in } \Omega_{2} \backslash \Omega_{1},\end{cases}
$$

almost everywhere. Hence, we can represent $u$ by $\mathbb{1}_{\Omega}$ for some Borel set $\Omega \in \mathbb{R}^{d}$ satisfying $\Omega_{1} \subset \Omega \subset \Omega_{2}$. Using the Cauchy inequality, we can estimate

$$
\begin{aligned}
J_{\epsilon_{n}, \Omega_{1}, \Omega_{2}, \alpha}\left(u_{n}\right) & \geq \int_{\mathbb{R}^{d}}\left[\frac{\epsilon_{n}\left|\nabla u_{h}\right|^{2}}{2}+\frac{1}{\epsilon_{n}} W\left(u_{n}\right)\right] \mathrm{d} x \quad\left(\text { because } W_{1} \geq W \text { and } W_{2} \geq W\right) \\
& \geq \int_{\mathbb{R}^{d}}\left[\frac{\epsilon_{n}\left|\nabla u_{n}\right|^{2}}{2}+\frac{1}{\epsilon_{n}} \tilde{W}\left(u_{n}\right)\right] \mathrm{d} x \quad\left(\text { where } \tilde{W}(s)=\min \left\{W(s) ; \sup _{s \in[0,1]} W(s)\right\}\right) \\
& \geq \int_{\mathbb{R}^{d}} \sqrt{2 \tilde{W}\left(u_{n}\right)}\left|\nabla u_{n}\right| \mathrm{d} x=\int_{\mathbb{R}^{d}}\left|\nabla\left[\phi\left(u_{n}\right)\right]\right| \mathrm{d} x=\left|D\left[\phi\left(u_{n}\right)\right]\right|\left(\mathbb{R}^{d}\right),
\end{aligned}
$$

where $\phi(s)=\int_{0}^{s} \sqrt{2 \tilde{W}(t)} d t$. Since $\phi$ is a Lipschitz function (because $\tilde{W}$ is bounded), $\phi\left(u_{\epsilon}\right)$ converges in $L^{1}\left(\mathbb{R}^{d}\right)$ to $\phi(u)$. Using the lower semicontinuity of $v \rightarrow|D v|\left(\mathbb{R}^{d}\right)$, we obtain

$$
\lim _{n \rightarrow+\infty} J_{\epsilon_{n}, \Omega_{1}, \Omega_{2}}\left(u_{n}\right) \geq \liminf _{n \rightarrow+\infty}\left|D \phi\left(u_{n}\right)\right|\left(\mathbb{R}^{d}\right) \geq|D \phi(u)|\left(\mathbb{R}^{d}\right) .
$$

The liminf inequality is finally obtained remarking that $\phi(u)=\phi\left(\mathbb{1}_{\Omega}\right)=c_{W} \mathbb{1}_{\Omega}=c_{W} u$.

Let us now prove the limsup inequality. 
(ii) Limsup inequality:

We first assume that $u=\mathbb{1}_{\Omega}$ for some bounded open set $\Omega$ satisfying $\Omega_{1} \subset \Omega \subset \Omega_{2}$ with smooth boundaries. We introduce the sequence

$$
u_{\epsilon}(x)=q\left(\frac{\operatorname{dist}(x, \Omega)}{\epsilon}\right) .
$$

and two constants $c_{1}$ and $c_{2}$ defined by

$$
c_{1}=\sup _{s \in[0,1]}\left\{W_{1}(s)-W(s)\right\}, \quad \text { and } \quad c_{2}=\sup _{s \in[0,1]}\left\{W_{2}(s)-W(s)\right\} .
$$

Note that

$$
\begin{aligned}
J_{\epsilon, \Omega_{1}, \Omega_{2}, \alpha}\left(u_{\epsilon}\right)= & \int_{\mathbb{R}^{d}}\left[\frac{\epsilon\left|\nabla u_{\epsilon}\right|^{2}}{2}+\frac{1}{\epsilon} W\left(u_{\epsilon}\right)\right] \mathrm{d} x+\int_{\mathbb{R}^{d}} \frac{1}{\epsilon} q\left(\frac{\operatorname{dist}\left(x, \Omega_{1}\right)}{\epsilon^{\alpha}}\right)\left(W_{1}\left(u_{\epsilon}\right)-W\left(u_{\epsilon}\right)\right) \mathrm{d} x \\
& +\int_{\mathbb{R}^{d}} \frac{1}{\epsilon} q\left(\frac{\operatorname{dist}\left(x, \Omega_{2}^{c}\right)}{\epsilon^{\alpha}}\right)\left(W_{2}\left(u_{\epsilon}\right)-W\left(u_{\epsilon}\right)\right) \mathrm{d} x .
\end{aligned}
$$

Each of these 3 terms above is now analyzed.

(1) Estimation of the first term:

$$
\mathcal{I}_{\epsilon}^{1}=\int_{\mathbb{R}^{d}}\left[\frac{\epsilon\left|\nabla u_{\epsilon}\right|^{2}}{2}+\frac{1}{\epsilon} W\left(u_{\epsilon}\right)\right] \mathrm{d} x
$$

By co-area formula, we estimate

$$
\begin{aligned}
\mathcal{I}_{\epsilon}^{1} & =\frac{1}{\epsilon} \int_{\mathbb{R}^{d}}\left[\frac{q^{\prime}(d(x, \Omega) / \epsilon)^{2}}{2}+W(q(d(x, \Omega) / \epsilon))\right] \mathrm{d} x \\
& =\frac{1}{\epsilon} \int_{\mathbb{R}} g(s)\left[\frac{q^{\prime}(s / \epsilon)^{2}}{2}+W(q(s / \epsilon))\right] \mathrm{d} s \\
& =\int_{\mathbb{R}} g(\epsilon t)\left[\frac{q^{\prime}(t)^{2}}{2}+W(q(t))\right] \mathrm{d} t
\end{aligned}
$$

where $g(s)=\left|D \mathbb{1}_{\{d \leq s\}}\right|\left(\mathbb{R}^{d}\right)$.

By the smoothness of $\partial \Omega, g(\epsilon t)$ converges to $\left|D \mathbb{1}_{\{\operatorname{dist}(x, \Omega) \leq 0\}}\right|\left(R^{d}\right)$ as $\epsilon \rightarrow 0$; moreover, by definition of the profile $q, u_{\epsilon}$ converges to $\mathbb{1}_{\Omega}$ and

$$
\limsup _{\epsilon \rightarrow 0} \mathcal{I}_{\epsilon}^{1} \leq\left|D \mathbb{1}_{\Omega}\right|\left(\mathbb{R}^{d}\right) \int_{-\infty}^{+\infty}\left[\frac{1}{2}\left|q^{\prime}(s)\right|^{2}+W(q(s)) \mathrm{d} s\right] .
$$

According to Remark (1.1), it follows that

$$
\int_{-\infty}^{+\infty}\left[\frac{1}{2}\left|q^{\prime}(s)\right|^{2}+W(q(s))\right] \mathrm{d} s=\int_{0}^{1} \sqrt{2 W(s)} \mathrm{d} s=c_{W},
$$

which implies that

$$
\limsup _{\epsilon \rightarrow 0} \mathcal{I}_{\epsilon}^{1} \leq c_{W}\left|D \mathbb{1}_{\Omega}\right|\left(\mathbb{R}^{d}\right)
$$

(2) Estimation of the second term:

$$
\mathcal{I}_{\epsilon}^{2}=\int_{\mathbb{R}^{d}} \frac{1}{\epsilon} q\left(\frac{\operatorname{dist}\left(x, \Omega_{1}\right)}{\epsilon^{\alpha}}\right)\left(W_{1}\left(u_{\epsilon}\right)-W\left(u_{\epsilon}\right)\right) \mathrm{d} x .
$$

The function $\operatorname{dist}(x, \Omega)$ is negative on $\Omega_{1}$, thus $u_{\epsilon}(x) \geq \frac{1}{2}$ on $\Omega_{1}$ and $W_{1}\left(u_{\epsilon}(x)\right)=W\left(u_{\epsilon}(x)\right)$ for all $x \in \Omega_{1}$. 
This means that

$$
\mathcal{I}_{\epsilon}^{2}=\int_{\mathbb{R}^{d} \backslash \Omega_{1}} \frac{1}{\epsilon} q\left(\frac{\operatorname{dist}\left(x, \Omega_{1}\right)}{\epsilon^{\alpha}}\right)\left(W_{1}\left(u_{\epsilon}\right)-W\left(u_{\epsilon}\right)\right) \mathrm{d} x \leq c_{1} \int_{\mathbb{R}^{d} \backslash \Omega_{1}} \frac{1}{\epsilon} q\left(\frac{\operatorname{dist}\left(x, \Omega_{1}\right)}{\epsilon^{\alpha}}\right) \mathrm{d} x,
$$

where $c_{1}=\sup _{s \in[0,1]}\left\{W_{1}(s)-W(s)\right\}$.

Using co-area formula, we estimate

$$
\int_{\mathbb{R}^{d} \backslash \Omega_{1}} \frac{1}{\epsilon} q\left(\frac{\operatorname{dist}\left(x, \Omega_{1}\right)}{\epsilon^{\alpha}}\right) \mathrm{d} x=\int_{0}^{\infty} \frac{1}{\epsilon} g_{1}(s) q\left(\frac{s}{\epsilon^{\alpha}}\right) \mathrm{d} s=\epsilon^{\alpha-1} \int_{0}^{\infty} g_{1}\left(\epsilon^{\alpha} s\right) q(s) \mathrm{d} s,
$$

where $g_{1}(s)=\left|D \mathbb{1}_{\left\{\operatorname{dist}\left(x, \Omega_{1}\right) \leq s\right\}}\right|\left(\mathbb{R}^{d}\right)$.

By the smoothness of $\Omega_{1}, g\left(\epsilon^{\alpha} t\right)$ converges to $\left|D \mathbb{1}_{\text {dist }\left(x, \Omega_{1}\right) \leq 0}\right|\left(R^{d}\right)$ as $\epsilon \rightarrow 0$. We then deduce that

$$
\limsup _{\epsilon \rightarrow 0} \mathcal{I}_{\epsilon}^{2}=0
$$

as $\alpha>1$ and $\int_{0}^{\infty} q(s) \mathrm{d} s$ is bounded.

(3) Estimation of the last term:

$$
\mathcal{I}_{\epsilon}^{3}=\int_{\mathbb{R}^{d}} \frac{1}{\epsilon} q\left(\frac{\operatorname{dist}\left(x, \Omega_{2}^{c}\right)}{\epsilon^{\alpha}}\right)\left(W_{2}\left(u_{\epsilon}\right)-W\left(u_{\epsilon}\right)\right) \mathrm{d} x .
$$

This estimation is similar to the second one. The function $\operatorname{dist}(x, \Omega)$ is positive on $\mathbb{R}^{d} \backslash \Omega_{2}$, this means $u_{\epsilon}(x) \leq \frac{1}{2}$ on $\mathbb{R}^{d} \backslash \Omega_{2}$ and $W_{2}\left(u_{\epsilon}(x)\right)=W\left(u_{\epsilon}(x)\right)$ for all $x \in \mathbb{R}^{d} \backslash \Omega_{2}$. Then, we have

$$
\mathcal{I}_{\epsilon}^{3}=\int_{\Omega_{2}} \frac{1}{\epsilon} q\left(\frac{\operatorname{dist}\left(x, \Omega_{2}^{c}\right)}{\epsilon^{\alpha}}\right)\left(W_{2}\left(u_{\epsilon}\right)-W\left(u_{\epsilon}\right)\right) \mathrm{d} x \leq c_{2} \int_{\Omega_{2}} \frac{1}{\epsilon} q\left(\frac{\operatorname{dist}\left(x, \Omega_{2}^{c}\right)}{\epsilon^{\alpha}}\right) \mathrm{d} x,
$$

and using co-area formula, it holds

$$
\int_{\Omega_{2}} \frac{1}{\epsilon} q\left(\frac{\operatorname{dist}\left(x, \Omega_{2}^{c}\right)}{\epsilon^{\alpha}}\right) \mathrm{d} x=\int_{0}^{\infty} \frac{1}{\epsilon} g_{2}(s) q\left(\frac{s}{\epsilon^{\alpha}}\right) \mathrm{d} s=\epsilon^{\alpha-1} \int_{0}^{\infty} g_{2}\left(\epsilon^{\alpha} s\right) q(s) \mathrm{d} s,
$$

where $g_{2}(s)=\left|D \mathbb{1}_{\left\{\operatorname{dist}\left(x, \Omega_{2}\right) \leq-s\right\}}\right|\left(\mathbb{R}^{d}\right)$. We deduce as before that

$$
\limsup _{\epsilon \rightarrow 0} \mathcal{I}_{\epsilon}^{3}=0
$$

Finally, we conclude that

$$
\limsup _{\epsilon \rightarrow 0} J_{\epsilon, \Omega_{1}, \Omega_{2}, \alpha}\left(u_{\epsilon}\right) \leq c_{W}\left|D \mathbb{1}_{\Omega}\right|\left(\mathbb{R}^{d}\right) .
$$

Remark 3.3. This theorem is still true in the limit case $\alpha \rightarrow \infty$, where $J_{\epsilon, \Omega_{1}, \Omega_{2}, \alpha=\infty}(u)$ reads

$$
J_{\epsilon, \Omega_{1}, \Omega_{2}, \infty}(u)=\int_{\Omega_{1}}\left[\frac{\epsilon|\nabla u|^{2}}{2}+\frac{1}{\epsilon} W_{1}(u)\right] \mathrm{d} x+\int_{\Omega_{2} \backslash \Omega_{1}}\left[\frac{\epsilon|\nabla u|^{2}}{2}+\frac{1}{\epsilon} W(u)\right] \mathrm{d} x+\int_{\mathbb{R}^{d} \backslash \Omega_{2}}\left[\frac{\epsilon|\nabla u|^{2}}{2}+\frac{1}{\epsilon} W_{2}(u)\right] .
$$

\subsection{Some remarks about sharp interface limit of the gradient flow}

We have just etablished a connection between the penalized perimeter $J_{\Omega_{1}, \Omega_{2}}$ and its smooth approximation $J_{\epsilon, \Omega_{1}, \Omega_{2}, \alpha}$. In Section 4, we will introduce a numerical scheme associated to the penalized Allen Cahn (2.3) to approximate the mean curvature flow with obstacle defined as the gradient flow of $J_{\Omega_{1}, \Omega_{2}}$. 
A first remark concerns the geometric evolution of a mean curvature flow with obstacle $\Omega(t) \subset \mathbb{R}^{d}, 0 \leq t \leq T$, from a smooth domain $\Omega_{1} \subset \Omega_{0} \subset \Omega_{2}$. The outward normal velocity at point $x \in \partial \Omega(t)$ satisfies for regular interfaces

$$
V_{n}(x)= \begin{cases}\kappa(x) & \text { if } x \in \Omega_{2} \backslash \Omega_{1} \\ \max \{\kappa(x), 0\} & \text { if } x \in \partial \Omega_{1} \\ \min \{\kappa(x), 0\} & \text { if } x \in \partial \Omega_{2},\end{cases}
$$

where $\kappa$ denotes the mean curbature at the interface. Some recent results about existence, unicity and $C^{1,1}$ regularity of such flow have been established in [3] for smooth obstacles. Notice also that in this case, $V_{n}$ is discontinous on $\partial \Omega$, then the classical viscosity theory [21] does no apply. The existence of motion in general sense (when singularies appear) is still an open issue to our knowledge.

A second remark concerns the gradient flow of $J_{\epsilon, \Omega_{1}, \Omega_{2}, \alpha}$ defined by

$$
u_{t}=\triangle u-\frac{1}{\epsilon^{2}} \partial_{s} W_{\epsilon, \Omega_{1}, \Omega_{2}, \alpha}(u, x) \text {. }
$$

Since the the potential $(s, x) \rightarrow W_{\epsilon, \Omega_{1}, \Omega_{2}, \alpha}(s, x)$ is smooth, the proof of existence, unicity and comparison principle can be easily derived from the original Allen Cahn equation properties.

Finally, the global result of convergence of the gradient flow of $J_{\epsilon, \Omega_{1}, \Omega_{2}, \alpha}$ to $J_{\Omega_{1}, \Omega_{2}}$ is a difficult problem for which classical approaches can not apply directly.

A first way would be to used the recent theory of Serfaty [40] which insures the $\Gamma$-convergence of the gradient flow associated to a family of energy. In the case of Allen Cahn equation, the assumptions needed in this theory are relied to the De Giorgi conjecture about the gamma-convergence of

$$
F_{\epsilon}(u)=\epsilon \int_{\mathbb{R}^{d}}\left(\Delta u-\frac{1}{\epsilon^{2}} W^{\prime}(u)\right)^{2} \mathrm{~d} x,
$$

to Willmore energy

$$
F(\Omega)=\int_{\partial \Omega} \kappa^{2} \mathrm{~d} \sigma(x)
$$

This conjecture has been recently established [37-39], but only in the case of $C^{2}$ interfaces, which does not correspond to the regularity of mean curvature flow with obstacles.

Other approachs $[10,17]$ should be more suitable but need a deeper analysis. Indeed, the proofs are based on a comparison principle which is still satisfied by our modified Allen Cahn equation, and an explicit construction of a subsolution and supsolution. Unfortunately, such constructions also need a $C^{2}$ regularity of the interface.

\section{Algorithms And numerical Simulations}

We now compare numerically the two phase field models described in Section 2. The first and classical model is integrated by a semi-implicit finite element method whereas our penalized Allen Cahn equation is solved by the semi-implicit Fourier spectral algorithm. In particular, we will observe that both approaches give similar solutions but, the convergence rate of the phase field approximation appears to behave as about $O(\epsilon \ln (\epsilon))$ for the Dirichlet model and as $O\left(\epsilon^{2} \ln (\epsilon)^{2}\right)$ (when $\alpha$ is sufficiently large) for our penalized version of Allen Cahn equation.

\subsection{A semi-implicit finite element method for the Allen Cahn equation with Dirichlet boundary conditions}

Let us give more precision about the classic semi-implicit finite element method used for the equation

$$
u_{t}(x, t)=\Delta u(x, t)-\frac{1}{\epsilon^{2}} W^{\prime}(u)(x, t), \quad \text { on } \quad \Omega_{2} \backslash \Omega_{1} \times[0, T],
$$

where $u_{\mid \partial \Omega_{1}}=1, u_{\mid \partial \Omega_{2}}=0$ and $W(s)=\frac{1}{2} s^{2}(1-s)^{2}$. 
Note that when the initial condition $u_{0}$ is chosen of the form $u_{0}=q\left(\operatorname{dist}\left(\Omega_{0}, x\right) / \epsilon\right)$ with $\Omega_{0}$ satisfying the constraint $\Omega_{1} \subset \Omega_{0} \subset \Omega_{2}$, then we expect that the set $\Omega^{\epsilon}(t)$ defined by

$$
\Omega^{\epsilon}(t)=\Omega_{1} \cup\left\{x \in \Omega_{2} \backslash \Omega_{1} ; u(x, t) \geq 1 / 2\right\},
$$

should be a good approximation to the constrained mean curvature flow $t \rightarrow \Omega(t)$.

Let us introduce a triangulation mesh $\mathcal{T}_{h}$ on the set $\Omega_{2} \backslash \Omega_{1}$ and the discretization time step $\delta_{t}$. Then, we consider the approximation spaces $X_{h, 0}$ and $X_{h}$ defined by

$$
\left\{\begin{array}{l}
X_{h}=\left\{v \in H^{1}\left(\overline{\Omega_{2} \backslash \Omega_{1}}\right) \cup C^{0}\left(\overline{\Omega_{2} \backslash \Omega_{1}}\right) ; v_{\mid K \in \mathcal{T}_{h}} \in P_{k}(K), \quad v_{\mid \Omega_{1}}=1 \text { and } v_{\mid \Omega_{2}}=0\right\} \\
X_{h, 0}=\left\{v \in H_{0}^{1}\left(\Omega_{2} \backslash \Omega_{1}\right) \cup C^{1}\left(\Omega_{2} \backslash \Omega_{1}\right) ; v_{\mid K \in \mathcal{T}_{h}} \in P_{2}(K)\right\}
\end{array}\right.
$$

where $P_{k}$ denotes the polynomial space of degree $k$. We take $k=2$ in the future numerical illustrations. Then, the solution $u\left(x, t_{n}\right)$ at time $t_{n}=n \delta_{t}$ is approximated by $U^{h, n}$, defined for $n>1$ as the solution on $X_{h}$ of

$$
\int_{\Omega_{2} \backslash \Omega_{1}} U^{h, n} \varphi \mathrm{d} x+\delta_{t} \int_{\Omega_{2} \backslash \Omega_{1}} \nabla U^{h, n} \nabla \varphi \mathrm{d} x=\int_{\Omega_{2} \backslash \Omega_{1}}\left(U^{h, n-1}-\frac{\delta_{t}}{\epsilon^{2}} W^{\prime}\left(U^{h, n-1}\right)\right) \varphi \mathrm{d} x, \quad \forall \varphi \in X_{h, 0},
$$

and for $n=0$ by

$$
U^{h, 0}=\underset{v \in X_{h}}{\arg \min }\left\|v-u_{0}\right\|_{L^{2}\left(\Omega_{2} \backslash \Omega_{1}\right)} .
$$

This algorithm is known to be stable under the condition

$$
\delta_{t} \leq c_{W} \epsilon^{2},
$$

where $c_{W}=\left[\sup _{t \in[0,1]}\left\{W^{\prime \prime}(s)\right\}\right]^{-1}$. More results about stability and convergence of finite element method for the resolution of Allen Cahn equation can be found in [13,26-28, 42].

\subsection{A time-splitting Fourier spectral method for the penalized Allen-Cahn equation}

We now consider the second model

$$
u_{t}(x, t)=\triangle u(x, t)-\frac{1}{\epsilon^{2}} \partial_{s} W_{\epsilon, \Omega_{1}, \Omega_{2}, \alpha}(u(x, t), x), \quad \text { on } \quad Q \times[0, T],
$$

with periodic boundary conditions on a given box $Q$, chosen sufficiently large to contain $\Omega_{2}$. In future numerical tests, we use $\alpha=2, W(s)=\frac{1}{2} s^{2}(1-s)^{2}$ and the potentials $W_{1}, W_{2}$ are defined by

$$
W_{1}(s)=\left\{\begin{array}{ll}
\frac{1}{2} s^{2}(1-s)^{2} & \text { if } s \geq \frac{1}{2} \\
10(s-0.5)^{4}+1 / 32 & \text { otherwise }
\end{array} \quad \text { and } \quad W_{2}(s)= \begin{cases}\frac{1}{2} s^{2}(1-s)^{2} & \text { if } s \leq \frac{1}{2} \\
10(s-0.5)^{4}+1 / 32 & \text { otherwise }\end{cases}\right.
$$

which clearly satisfy the assumption $(H 1)$ (see Fig. 2).

The initial condition $u_{0}$ satisfies $u_{0}=q\left(\operatorname{dist}\left(\Omega_{0}, x\right) / \epsilon\right)$ and we will show that the set

$$
\Omega^{\epsilon}(t)=\{x \in Q ; u(x, t) \geq 1 / 2\},
$$

will be a good approximation of $\Omega(t)$ as $\epsilon$ tends to zero.

Our numerical scheme for solving equation (4.2) is based on a splitting method between the diffusion and reaction terms. We take advantage of the periodicity of $u$ by integrating exactly the diffusion term in the Fourier space. More precisely, the solution $u\left(x, t_{n}\right)$ at time $t_{n}=t_{0}+n \delta_{t}$ is approximated by its truncated Fourier series:

$$
u_{P}^{n}(x)=\sum_{|p|_{\infty}=P} c_{p}^{n} \mathrm{e}^{2 i \pi p \cdot x} .
$$

Here $|p|_{\infty}=\max _{1 \leq i \leq d}\left|p_{i}\right|$ and $P$ represents the number of Fourier modes in each direction. 


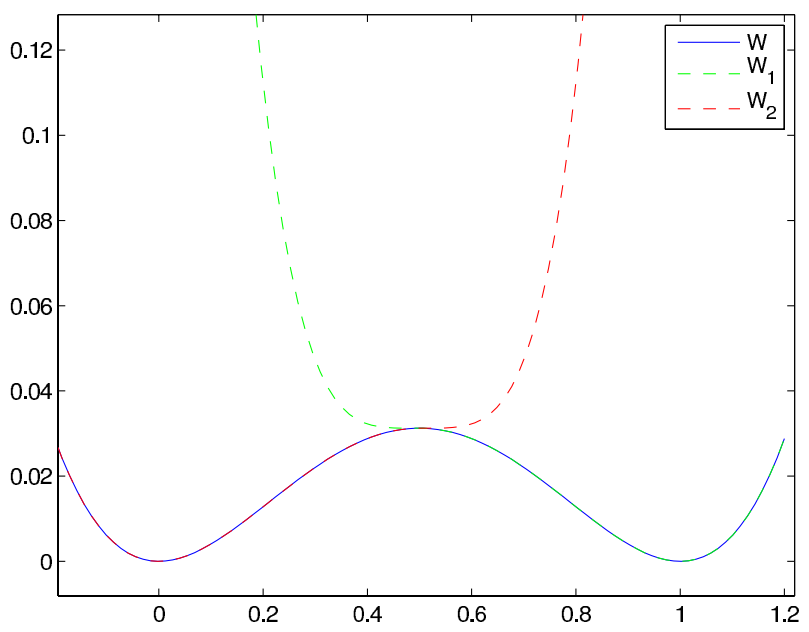

Figure 2. Example of potentials $W, W_{1}$ and $W_{2}$.

The step $n$ of our algorithm writes:

- $u_{P}^{n+1 / 2}(x)=\sum c_{p}^{n+1 / 2} \mathrm{e}^{2 i \pi p \cdot x}, \quad$ with $\quad c_{p}^{n+1 / 2}=c_{p}^{n} \mathrm{e}^{-4 \pi^{2} \delta t|p|^{2}}$

- $u_{P}^{n+1}=u_{P}^{n+1 / 2}-\frac{\delta t}{\epsilon^{2}} \partial_{s} W_{\epsilon, \Omega_{2}, \Omega_{1}, \alpha}\left(u_{P}^{n+1 / 2}, x\right)$.

In practice, the first step is performed via a Fast Fourier Transform, with a computational cost $O\left(P^{d} \ln (P)\right)$. In the simplest case of traditional Allen Cahn equation, the corresponding numerical scheme turns out to be stable under the condition

$$
\delta t \leq c_{W} \epsilon^{2}
$$

and the convergence of this splitting approach has been established in [15]. In practice, the parameter $\epsilon$ has to be chosen great than the spatial discretisation step $\delta_{x}=1 / P$.

\subsection{Simulations and numerical convergence}

We compare in this part numerical solutions obtained with these two algorithms. For each test, we took $\epsilon=2^{-8}$ and $\delta_{t}=\epsilon^{2}$. The $P_{2}$ finite element algorithm was implemented in Freefem ++ . The mesh $\mathcal{T}_{h}$ used in these simulations are plotted in Figure 3. The penalization method was implemented in MATLAB where we have taken $P=2^{8}$.

We first plot two situations in Figures 4 and 5 . The functions $u_{\epsilon}$ are plotted only on the admissible set $\Omega_{2} \backslash \Omega_{1}$ for the FE Dirichlet method and on all the set $Q$ for the pseudo-spectral penalization version with $\alpha=2$. We note that the solutions obtained by both methods are very similar.

In order to estimate the convergence rate of both models, we consider the case where $\Omega_{1}$ and $\Omega_{2}$ are two circles of radii equal to $R_{1}=0.3$ and $R_{2}=0.4$. The situation is thus very simple when the initial set $\Omega_{0}$ is also a circle with radius $R_{0}$ satisfying $R_{1}<R_{0}<R_{2}$. Indeed, the penalized mean curvature motion $\Omega(t)$ evolves as a circle, with radius satisfying

$$
R(t)=\max \left(\sqrt{R_{0}^{2}-2 t}, R_{1}\right)
$$

that decreases until $R(t)=R_{1}$.

The solutions of the two different models are computed for different values of $\epsilon$ with $P=2^{8}, \delta_{t}=1 / P^{2}$ and $R_{0}=0.35$. In both cases, the set $\Omega^{\epsilon}(t)$ appears as a circle of radius $R^{\epsilon}(t)$. We then estimate the numerical 

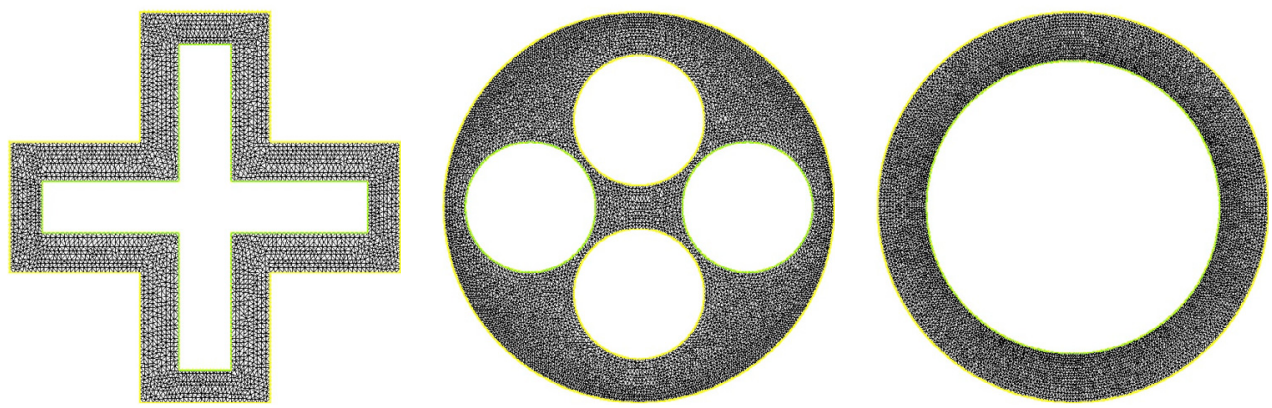

Figure 3. Mesh $\mathcal{T}_{h}$ generated by Freefem++, and used in simulations plotted in Figures 4 and 5 . In both cases, $\partial \Omega_{1}$ and $\partial \Omega_{2}$ are respectively identified as the green and the yellow boundaries.
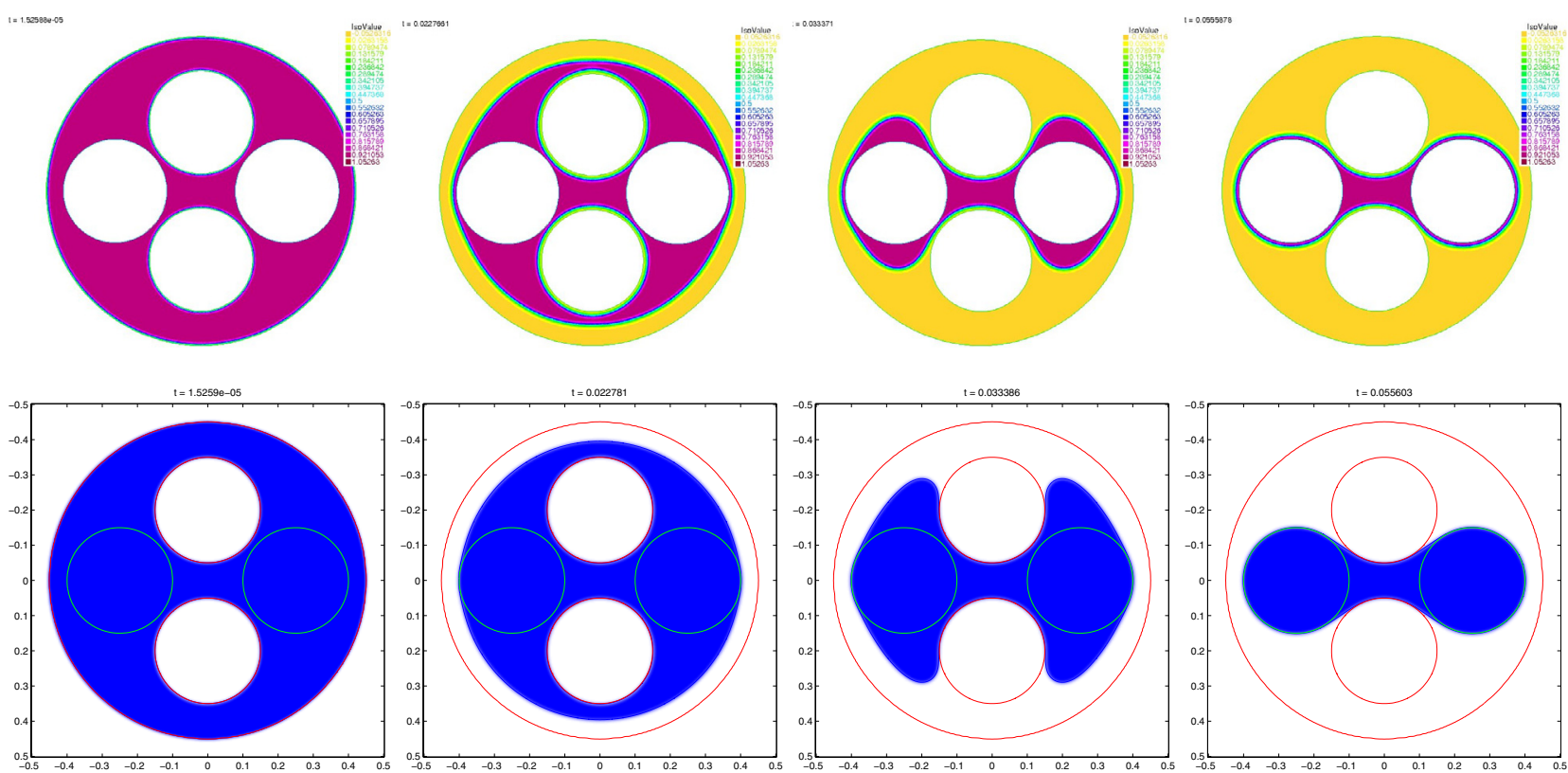

FiguRE 4. Numerical solutions obtained at different times $t_{0}=0, t_{1}=0.022, t_{2}=0.033$ and $t=0.055$. The first line corresponds to the FE Dirichlet method and the second line to the penalization pseudo-spectral approach.

error between $R^{\epsilon}(t)$ and $R(t)$. The results obtained for the first method are plotted in Figure 6: the first figure corresponds to the evolution $t \rightarrow \mathbb{R}^{\epsilon}(t)$ for 4 different values of $\epsilon$ and the second figure shows the error

$$
\epsilon \rightarrow \sup _{t \in[0, T]}\left\{\left|R(t)-R^{\epsilon}(t)\right|\right\}
$$

in logarithmic scale. It clearly appears an error of $O(\epsilon \ln (\epsilon))$.

The same test is done for the penalization algorithm with $\alpha=2$ : the results are plotted in Figure 7 and we now clearly observed a convergence rate of $O\left(\epsilon^{2} \ln \left(\epsilon^{2}\right)\right)$.

Remark 4.1 (about violation of the constraint $\Omega_{1} \subset \Omega \subset \Omega_{2}$ ). We remark that with our penalized Allen Cahn approach, the constraint $\Omega_{1} \subset \Omega \subset \Omega_{2}$ is always violated. Indeed, Figure 7 left shows that for a given $\epsilon$, 

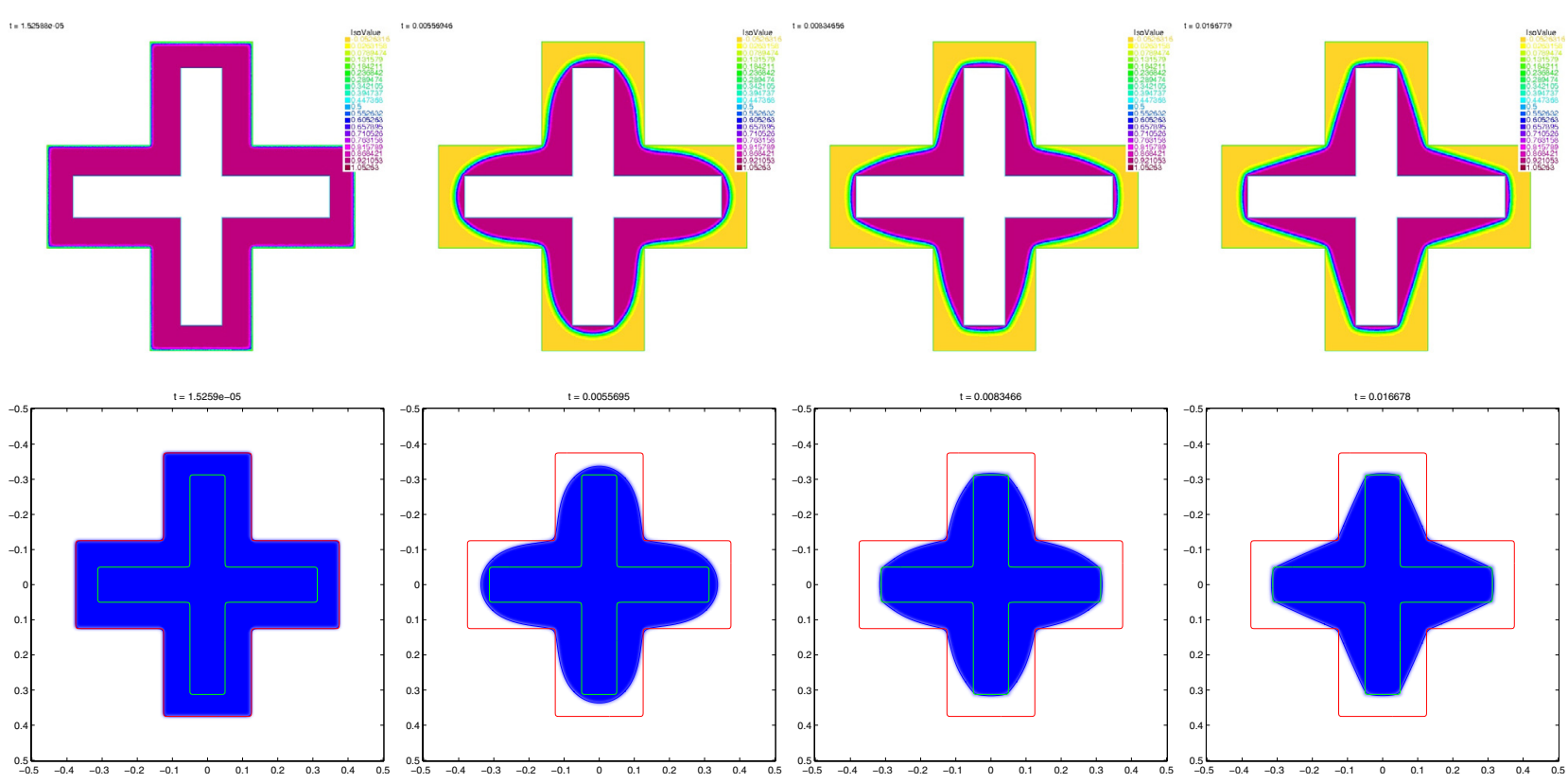

FigURE 5. Numerical solutions obtained at different times $t_{0}=0, t_{1}=0.0055, t_{2}=0.0083$ and $t_{3}=0.016$. The first line corresponds to the FE Dirichlet method and the second line to the penalization pseudo-spectral approach.
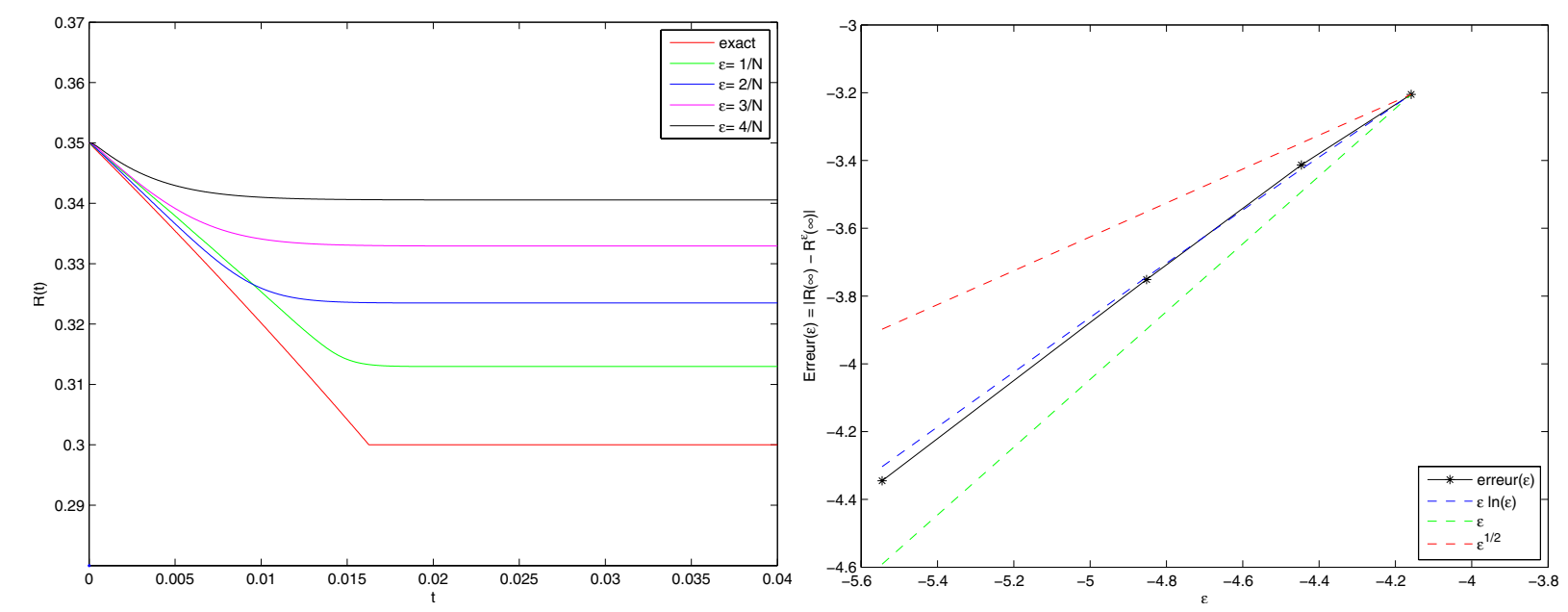

FiguRE 6. Dirichlet algorithm: numerical error $\left|R^{\epsilon}(t)-R(t)\right|$; left: $t \rightarrow R^{\epsilon}(t)$ for different values of $\epsilon$; right: $\epsilon \rightarrow \sup _{t \in[0, T]}\left\{\left|R(t)-R^{\epsilon}(t)\right|\right\}$ in logarithmic scale compare to curve slope $\sqrt{\epsilon}$ (red), $\epsilon$ (green) and $\epsilon \ln (\epsilon)$ (blue).

the radii of the circle $\Omega(t)$ converge in time to a value lower than the radius of $\Omega_{1}$. In fact, this error on the constraint appears only with an order of $O\left(\epsilon^{2} \ln \left(\epsilon^{2}\right)\right)$ as $\epsilon$ tends to zero. Moreover, the approximation of mean curvature flow by Allen Cahn equation is also of order $O\left(\epsilon^{2} \ln \left(\epsilon^{2}\right)\right)[10,17]$. This means that the precision on the constraint $\Omega_{1} \subset \Omega \subset \Omega_{2}$ do not affect the initial precision of the approximation of phase field method. 

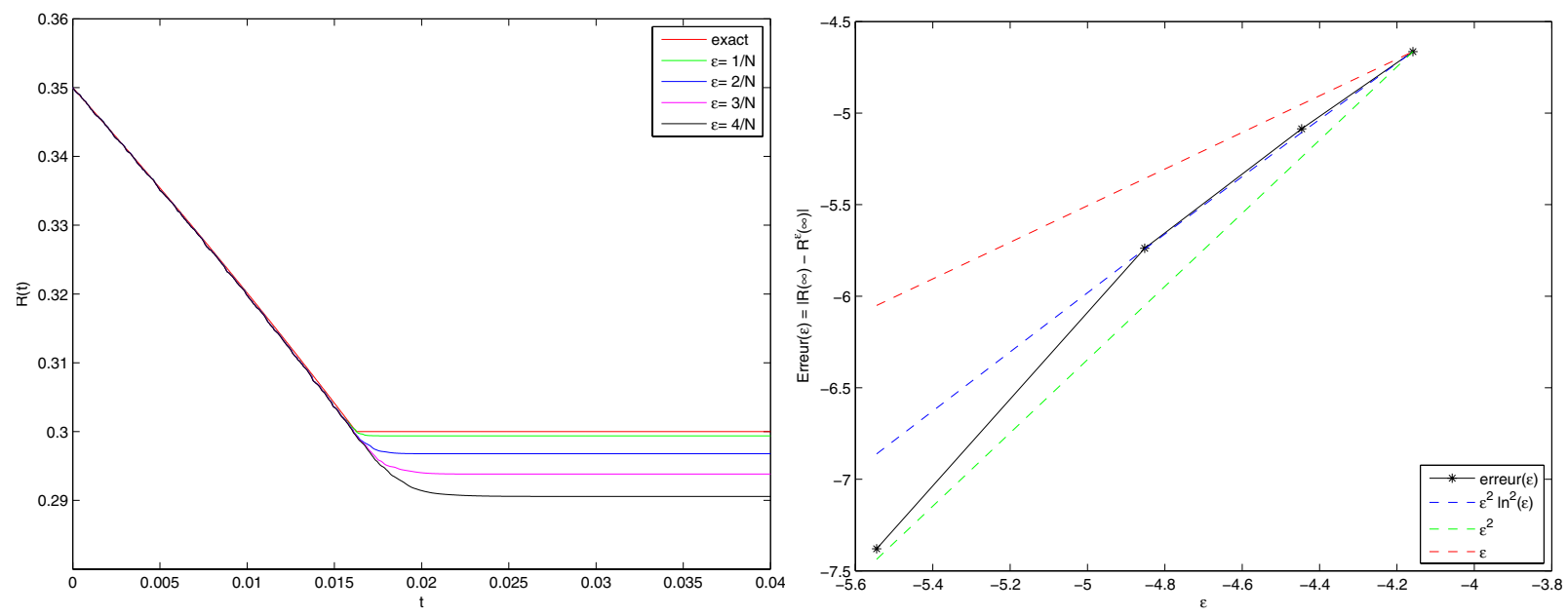

FiguRe 7. Penalization algorithm with $\alpha=2$ : numerical error $\left|R^{\epsilon}(t)-R(t)\right|$; left: $t \rightarrow R^{\epsilon}(t)$ for different values of $\epsilon$; right: $\epsilon \rightarrow \sup _{t \in[0, T]}\left\{\left|R(t)-R^{\epsilon}(t)\right|\right\}$ in logarithmic scale compare to curve of slope $\epsilon$ (red), $\epsilon^{2}$ (green) and $\epsilon^{2} \ln (\epsilon)^{2}$ (blue).
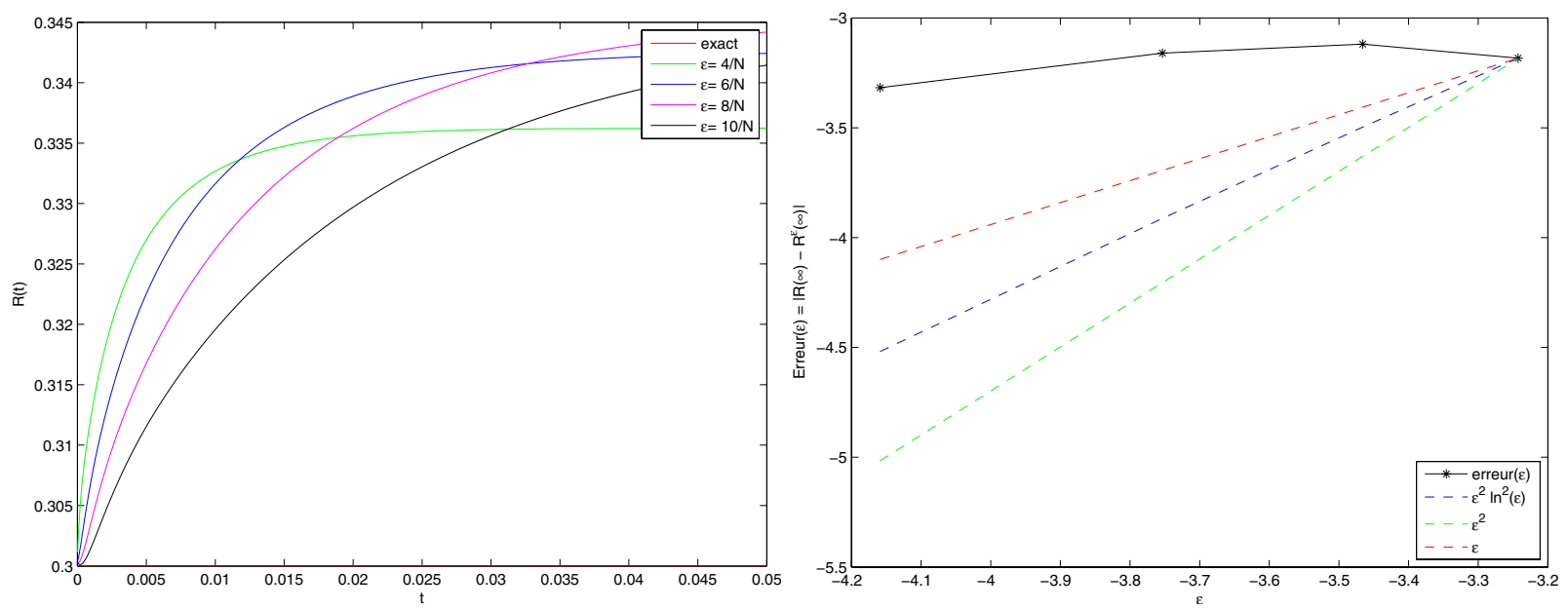

FiguRe 8. Penalization algorithm with $\alpha=1$ : numerical error $\left|R^{\epsilon}(t)-R(t)\right|$; left: $t \rightarrow R^{\epsilon}(t)$ for different values of $\epsilon$; right: $\epsilon \rightarrow \sup _{t \in[0, T]}\left\{\left|R(t)-R^{\epsilon}(t)\right|\right\}$ in logarithmic scale

In the following last test, we analyse numerically the impact of the coefficient $\alpha$ on our penalized Allen equation. As above, we consider the case where $\Omega_{1}$ and $\Omega_{2}$ are two circles of radii equal to $R_{1}=0.3$ and $R_{2}=0.4$. We consider as initial set $\Omega_{0}=\Omega_{1}$. The exact penalized mean curvature motion $\Omega(t)$ is then equal to $\Omega_{1}$ for all $t>0$.

Figure 8 shows experiment results obtained with $\alpha=1$. We clearly observe an error on the localization which does not decrease as $\epsilon$ tends to zero. This is consistant with our theorical analysis for which $\alpha$ is assumed to be strictly greater than 1 . Figure 9 presents the numerical errors for different values of alpha. We observe that the convergence rate is not affected by the value of $\alpha$, when $\alpha$ is chosen sufficiently far than 1 . In practice, we can also choose $\alpha=+\infty$, but this corresponds to use a discontinous potential $(s, x) \rightarrow W_{\epsilon, \Omega_{1}, \Omega_{2},+\infty}(s, x)$ in variable $x$. 


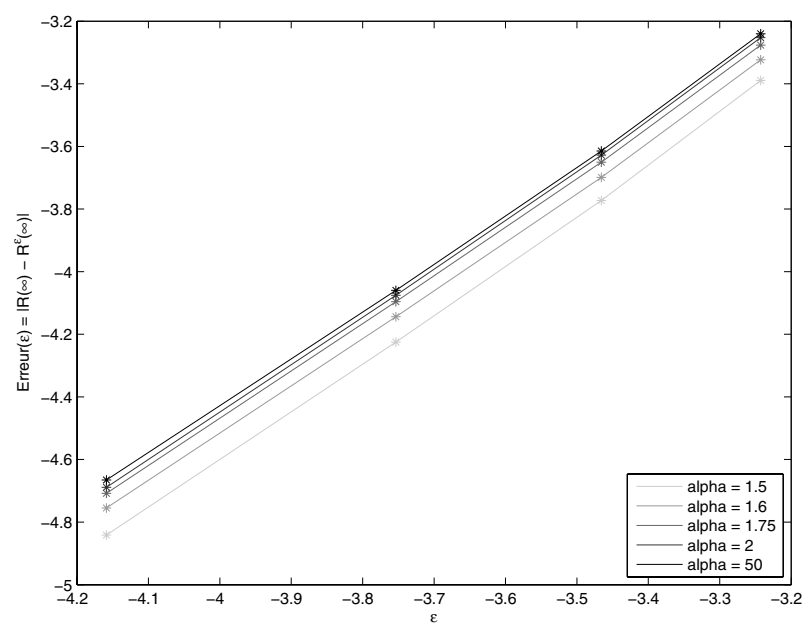

Figure 9. Penalization algorithm, numerical error $\left|R^{\epsilon}(t)-R(t)\right|$ for different values of $\alpha$; $\epsilon \rightarrow \sup _{t \in[0, T]}\left\{\left|R(t)-R^{\epsilon}(t)\right|\right\}$ in logarithmic scale.
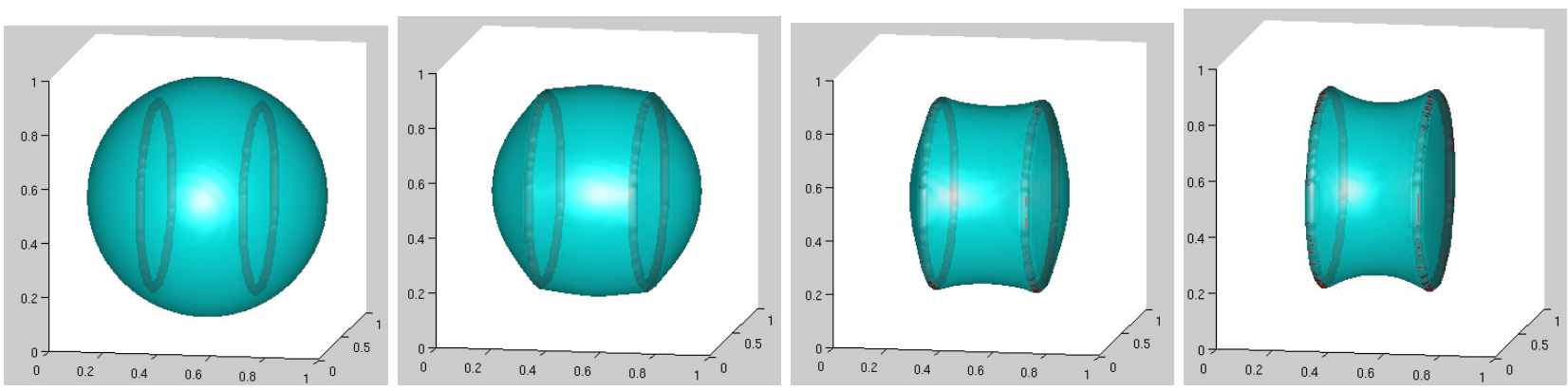

FigURE 10. Minimal surface estimation: solution of the Allen Cahn equation at different times $t$. The domain $\Omega_{1}$ is the union of the two red tori and $\Omega_{2}$ is the box $Q$ with $N=2^{7}, \epsilon=1 / N$ and $\delta_{t}=\epsilon^{2}$

Moreover, our approach allows us to simulate very easily and efficiently three dimensional experiments, whatever the geometry of the sets $\Omega_{1}$ and $\Omega_{2}$. See for instance Figure 10 where the numerical solution of the Allen Cahn equation is plotted for different times $t$.

\subsection{Possible extension of the method}

Another advantage of our penalization approach is that it can be easily extended for more general situations of evolving interfaces. For example, we have recently considered a mean curvature flow with an additional forcing term $g$ and a conservation of the volume in [14]. Then, the model of [14] can be modified to take into account additional inclusion-exclusion constraints by simply using potential $W_{\Omega_{1}, \Omega_{2}}$ instead of $W$ in phase field equation. In this case, this leads to the following perturbed Allen-Cahn equation

$$
u_{t}=\Delta u-\frac{1}{\epsilon^{2}} F(u),
$$

with

$$
F(u)=W_{\Omega_{1}, \Omega_{2}}^{\prime}(u)-\epsilon g \sqrt{2 W_{\Omega_{1}, \Omega_{2}}(u)}-\frac{\int_{Q} W_{\Omega_{1}, \Omega_{2}}^{\prime}(u)-\epsilon g \sqrt{2 W_{\Omega_{1}, \Omega_{2}}(u)} \mathrm{d} x}{\int_{Q} \sqrt{2 W_{\Omega_{1}, \Omega_{2}}(u)} \mathrm{d} x} \sqrt{2 W_{\Omega_{1}, \Omega_{2}}(u)} .
$$




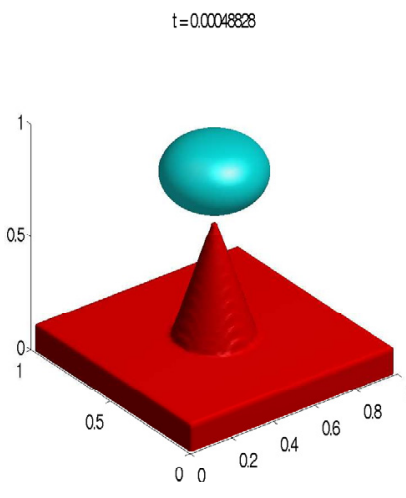

$t=0.00048828$

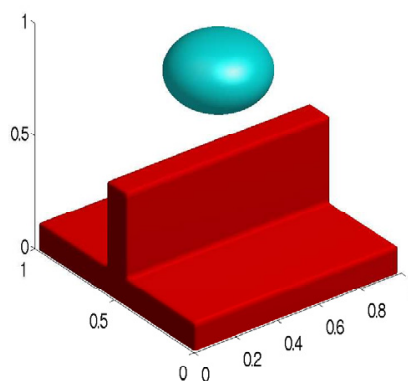

$t=0.050049$

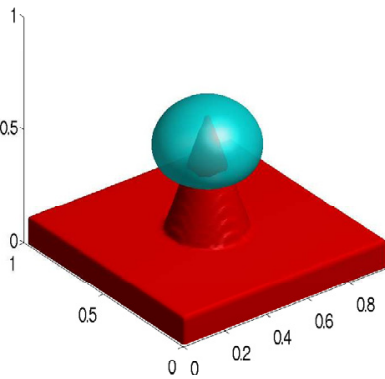

$t=0.0500049$

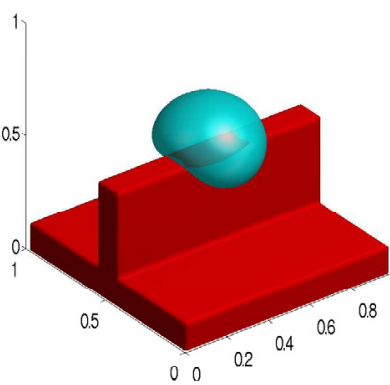

$t=0.090088$

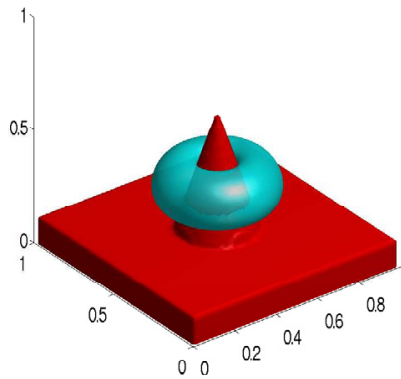

$t=0.0900088$

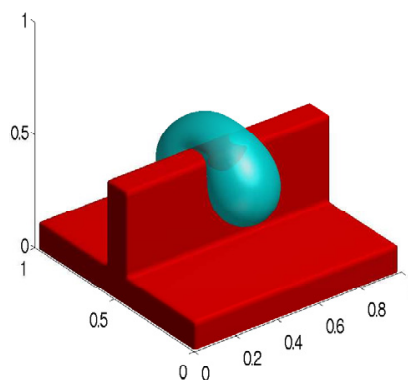

$t=0.1101$

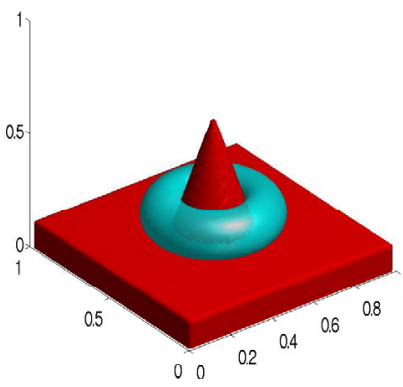

$t=0.11011$

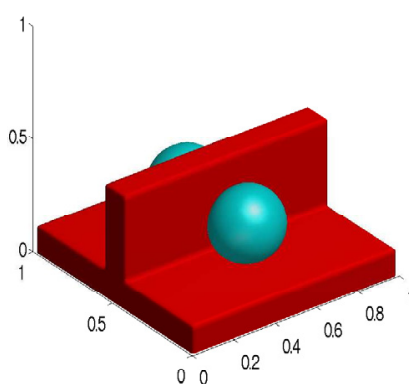

FiguRE 11. Two numerical experiments with a forcing term (gravity force), a volume conservation and an exclusion constraint: the domain $\Omega_{1}$ is empty and $\Omega_{2}$ is plotted in red in each picture. We used $N=2^{7}, \epsilon=1 / N$ and $\delta_{t}=\epsilon^{2}$.

Two simulations obtained from this model are plotted in Figure 11. We can observe that both constraints (conservation of the volume and inclusion-exclusion set) are well respected.

\section{Conclusion}

This paper has presented a new phase field model for the approximation of mean curvature flow with inclusionexclusion constraints. The classical method in such a situation is to solve the Allen-Cahn equation with Dirichlet boundary conditions. Since this method appears to be not optimal while its convergence is observed with a rate about $O(\epsilon \ln (\epsilon))$ only, we have introduced a new approach based on a penalized double well potential. This method was firstly motivated by a $\Gamma$-convergence result and secondly numerical tests suggesting that its convergence rate is about $O\left(\epsilon^{2} \ln (\epsilon)^{2}\right)$. The proof of the numerical precision of the scheme is still an open problem and will be the subject of a forthcoming paper. Another advantage of our method lies in its simplicity to be implemented, since it is only based on Fourier Transforms. This simplicity allows one to consider 3D Geometries, and to elaborate new strategies in more general situations, such as mean curvature flow with forcing term and conservation of volume.

\section{REFERENCES}

[1] G. Alberti, Variational models for phase transitions, an approach via $\gamma$-convergence, in Calculus of variations and partial differential equations (Pisa, 1996). Springer, Berlin (2000) 95-114.

[2] S.M. Allen and J.W. Cahn, A microscopic theory for antiphase boundary motion and its application to antiphase domain coarsening. Acta Metall. 27 (1979) 1085-1095. 
[3] L. Almeida, A. Chambolle and M. Novaga, Mean curvature flow with obstacle. Technical Report Preprint (2011).

[4] L. Ambrosio, Geometric evolution problems, distance function and viscosity solutions, in Calculus of variations and partial differential equations (Pisa, 1996). Springer, Berlin (2000) 5-93.

[5] G. Barles, Solutions de viscosité des équations de Hamilton-Jacobi, in Mathématiques 8 Applications (Berlin) [Mathematics E Applications]. Springer-Verlag, Paris 17 (1994).

[6] J.W. Barrett, H. Garcke and R. Nürnberg, On the parametric finite element approximation of evolving hypersurfaces in r3. J. Comput. Phys. 227 (2008) 4281-4307.

[7] J.W. Barrett, H. Garcke and R. Nürnberg, A variational formulation of anisotropic geometric evolution equations in higher dimensions. Numer. Math. 109 (2008) 1-44.

[8] P.W. Bates, S. Brown and J.L. Han, Numerical analysis for a nonlocal Allen-Cahn equation. Int. J. Numer. Anal. Model. 6 (2009) 33-49.

[9] G. Bellettini, Variational approximation of functionals with curvatures and related properties. J. Convex Anal. 4 (1997) 91-108.

[10] G. Bellettini and M. Paolini, Quasi-optimal error estimates for the mean curvature flow with a forcing term. Differ. Integral Equ. 8 (1995) 735-752.

[11] G. Bellettini and M. Paolini, Anisotropic motion by mean curvature in the context of Finsler geometry. Hokkaido Math. J. 25 (1996) 537-566.

[12] B. Bourdin and A. Chambolle, Design-dependent loads in topology optimization. ESAIM: COCV 9 (2003) $19-48$.

[13] M. Brassel, Instabilité de Forme en Croissance Cristalline. Ph.D. thesis, University Joseph Fourier, Grenoble (2008).

[14] M. Brassel and E. Bretin, A modified phase field approximation for mean curvature flow with conservation of the volume. Math. Meth. Appl. Sci. 34 (2011) 1157-1180.

[15] E. Bretin, Méthode de champ de phase et mouvement par courbure moyenne. Ph.D. thesis, Institut National Polytechnique de Grenoble (2009).

[16] A. Bueno-Orovio, V.M. Pérez-García and F.H. Fenton, Spectral methods for partial differential equations in irregular domains: The spectral smoothed boundary method. SIAM J. Sci. Comput. 28 (2006) 886-900.

[17] X. Chen, Generation and propagation of interfaces for reaction-diffusion equations. J. Differ. Equ. 96 (1992) $116-141$.

[18] L.Q. Chen and J. Shen, Applications of semi-implicit Fourier-spectral method to phase field equations. Comput. Phys. Commun. 108 (1998) 147-158.

[19] Y.G. Chen, Y. Giga and S. Goto, Uniqueness and existence of viscosity solutions of generalized mean curvature flow equations. Proc. Jpn Acad. Ser. A 65 (1989) 207-210.

[20] X.F. Chen, C.M. Elliott, A. Gardiner and J.J. Zhao, Convergence of numerical solutions to the Allen-Cahn equation. Appl. Anal. 69 (1998) 47-56.

[21] M.G. Crandall, H. Ishii and P.-L. Lions, User's guide to viscosity solutions of second order partial differential equations. Bull Amer. Math. Soc. 27 (1992) 1-68.

[22] K. Deckelnick and G. Dziuk, Discrete anisotropic curvature flow of graphs. ESAIM: M2AN 33 (1999) $1203-1222$.

[23] K. Deckelnick, G. Dziuk and C.M. Elliott, Computation of geometric partial differential equations and mean curvature flow. Acta Numer. 14 (2005) 139-232.

[24] L.C. Evans and J. Spruck, Motion of level sets by mean curvature I. J. Differ. Geom. 33 (1991) 635-681.

[25] L.C. Evans, H.M. Soner and P.E. Souganidis, Phase transitions and generalized motion by mean curvature. Commun. Pure Appl. Math. 45 (1992) 1097-1123.

[26] X. Feng and A. Prohl, Numerical analysis of the Allen-Cahn equation and approximation for mean curvature flows. Numer. Math. 94 (2003) 33-65.

[27] X. Feng and A. Prohl, Analysis of a fully discrete finite element method for the phase field model and approximation of its sharp interface limits. Math. Comput. 73 (2004) 541-567.

[28] X. Feng and H.-J. Wu, A posteriori error estimates and an adaptive finite element method for the Allen-Cahn equation and the mean curvature flow. J. Sci. Comput. 24 (2005) 121-146.

[29] Y. Li, H.G. Lee, D. Jeong and J. Kim, An unconditionally stable hybrid numerical method for solving the Allen-Cahn equation. Comput. Math. Appl. 60 (2010) 1591-1606.

[30] L. Modica and S. Mortola, Il limite nella $\Gamma$-convergenza di una famiglia di funzionali ellittici. Boll. Un. Mat. Ital. A 14 (1977) $526-529$.

[31] L. Modica and S. Mortola, Un esempio di $\Gamma^{-}$-convergenza. Boll. Un. Mat. Ital. B 14 (1977) 285-299.

[32] S. Osher and R. Fedkiw, Level Set Methods and Dynamic Implicit Surfaces, Springer-Verlag, New York. Appl. Math. Sci. (2002).

[33] S. Osher and N. Paragios, Geometric Level Set Methods in Imaging, Vision and Graphics. Springer-Verlag, New York (2003).

[34] S. Osher and J.A. Sethian, Fronts propagating with curvature-dependent speed: algorithms based on Hamilton-Jacobi formulations. J. Comput. Phys. 79 (1988) 12-49.

[35] N.C. Owen, J. Rubinstein and P. Sternberg, Minimizers and gradient flows for singularly perturbed bi-stable potentials with a dirichlet condition. Proc. R. Soc. London 429 (1990) 505-532. 
[36] M. Paolini, An efficient algorithm for computing anisotropic evolution by mean curvature, in Curvature flows and related topics, edited by Levico, 1994. Gakuto Int. Ser. Math. Sci. Appl. 5 (1995) 199-213.

[37] M. Röger and R. Schätzle, On a modified conjecture of De Giorgi. Math. Z. 254 (2006) 675-714.

[38] R. Schätzle, Lower semicontinuity of the Willmore functional for currents. J. Differ. Geom. 81 (2009) $437-456$.

[39] R. Schätzle, The Willmore boundary problem. Calc. Var. Partial Differ. Equ. 37 (2010) 275-302.

[40] S. Serfaty, Gamma-convergence of gradient flows on hilbert and metric spaces and applications. Disc. Cont. Dyn. Systems 31 (2011) 1427-1451.

[41] H.-C.Y. Yu, H.-Y. Chen and K. Thornton, Extended smoothed boundary method for solving partial differential equations with general boundary conditions on complex boundaries. Technical Report, arXiv:1107.5341v1 (2011). Submitted.

[42] J. Zhang and Q. Du, Numerical studies of discrete approximations to the Allen-Cahn equation in the sharp interface limit. SIAM J. Sci. Comput. 31 (2009) 3042-3063. 